\title{
Saccadic adaptation shapes perceived size: Common codes for action and perception
}

\author{
Alexandra Pressigout ${ }^{1} \cdot$ Céline Paeye $^{1} \cdot$ Karine Doré-Mazars ${ }^{1}$ \\ Published online: 28 July 2020 \\ (C) The Psychonomic Society, Inc. 2020
}

\begin{abstract}
Recent findings suggest that perceptual and motor systems share common codes; for instance, perceived object location is known to correlate with motor changes in the oculomotor system. Here, we investigate whether modifying saccade amplitude affects object size perception. Participants saw in peripheral vision a test disk that could vary in size across trials. This disk was then replaced by a small target cross, which was the signal to make a saccade. After the saccade, the target cross was extinguished and replaced by a reference disk (thus seen in foveal vision). Participants had to compare the post- to the pre-saccade disk sizes. Psychometric functions were obtained before and after one session of 142 saccades made toward the cross that either stepped toward the fixation point during the saccade (backward adaptation group) or remained stationary (control group). In the experimental group, stepping the target cross toward fixation during saccades decreased movement amplitude, a phenomenon called saccadic adaptation. We observed a concurrent shift in the psychometric functions reflecting a decrease in perceived object size. Such a perceptual modification did not occur in the control group. Our results reveal that motor changes co-occur with changes in perceived object size. Unlike previous studies evaluating the impact of saccadic adaptation on perceived location, we measured here the perception of another spatial feature (the object size) that is not relevant for the sensorimotor transformation. Theoretical implications of the strong links between oculomotor parameters and object perception are discussed.
\end{abstract}

Keywords Perception and action $\cdot$ Visual perception $\cdot$ Saccadic adaptation

Motor and perceptual representations determine the way we interact with the world (Markman \& Brendl, 2005). These interactions require the integration of different types of magnitude such as spatial, temporal, and numerical ones. The estimation of "how long," "how many times," and "how much" is vital for many decisions in our daily life (e.g., when crossing the road or grasping an object). Many studies are greatly interested in the idea that time, number, and space interact and share properties. A theory of magnitude (ATOM; Walsh, 2003) proposed that these dimensions are processed by a generalized magnitude system that presumably computes magnitudes according to a common metric (Winter, Marghetis, \& Matlock, 2015). This system is supposedly not restricted to the processing of time, number,

$\overline{\text { Alexandra Pressigout and Céline Paeye contributed equally to this work. }}$

Alexandra Pressigout

alexandra.pressigout@parisdescartes.fr

1 Vision Action Cognition Lab, University of Paris Institute of Psychology, Paris, France and space and could be involved in the processing of other perceptual dimensions such as size, luminance, or loudness, and more generally of all dimensions that can be represented along a continuous scale of increasing or decreasing magnitudes ("more" or "less"). Moreover, this model predicts that any modification in one dimension should affect the perceived magnitude of other dimensions in the same way — a prediction that has been supported by various behavioral studies (e.g., DeLong, 1981; Xuan, Zhang, He, \& Chen, 2007). For instance, the perceived duration of a stimulus increased with increasing stimulus numerosity, luminance, or size, which suggests that these magnitudes interact. The size-congruency effect (Henik \& Tzelgov, 1982; Kadosh et al., 2007) provides additional evidence that magnitudes processed by the generalized system interact with each other. Indeed, when participants had to judge which of two numbers is numerically larger, the physical font size of the numbers interfered with the numerical comparison itself. Responses were faster for congruent conditions (e.g., small numbers presented in small font) and slower for noncongruent conditions (e.g., small numbers presented in large font).

In the ATOM model, perceptual magnitudes are used for action. Obviously, grasping an object requires one to process 
its size. It should be noted that ATOM, because it focuses on short-time movements, is mainly concerned with the immediate sensorimotor consequences of processing magnitudes. For instance, number magnitude has been shown to affect pointing (Fischer, 2003; Hesse, Fiehler, \& Bremmer, 2016), swiping (Dotan \& Dehaene, 2013; Fischer, Fischer, Huber, Strauß, \& Moeller, 2018), and grasping movements (Badets \& Pesenti, 2010). In Fischer et al.'s study (2018), participants had to swipe an object to the left or to the right following the presentation of a number. The authors found that movement amplitude increased with number magnitude. Moreover, they observed the classical SNARC effect (spatial-numerical association of response codes; see also Dehaene, Bossini, \& Giraux, 1993) - that is, the movements were initiated faster to the left following a small number, and to the right following a large number. These interactions between space and quantity on motor properties have been observed not only for manual reaction times (Ren, Nicholls, Ma, \& Chen, 2011; Seegelke \& Wühr, 2018; Wühr \& Seegelke, 2018) but also for oculomotor responses (Fischer, Warlop, Hill, \& Fias, 2004; Myachykov, Cangelosi, Ellis, \& Fischer, 2015; Pressigout, Charvillat, Mersad, \& Doré-Mazars, 2018; Schwarz \& Keus, 2004; Pressigout \& Dore-Mazars, 2019). These studies support the claim that both action planning and action execution are influenced by different perceptual magnitudes. Overall, these behavioral data are consistent with the ATOM view that motor outputs reflect interactions between different perceptual dimensions.

At the neurological level, the perceptual and motor systems are intimately linked in the parietal cortex (Bueti \& Walsh, 2009; Culham \& Valyear, 2006; Piazza \& Eger, 2016). Therefore, because of this perceptual-motor link, and because the magnitudes of perceptual dimensions affect the magnitude of motor parameters, we can posit that the motor system could in turn affect perception. If so, a theory of a generalized system such as ATOM should encompass both perceptual and motor magnitudes, which should mutually influence each other rather than positing a unilateral impact of perceptual magnitudes on movement planning and/or execution.

Indeed, several lines of research emphasize the close link between perception and action (e.g., Creem-Regehr \& Kunz, 2010; Prinz, 1990): For instance, ideomotor theories state that action is inherently related to its perceivable effects (see Shin, Proctor, \& Capaldi, 2010, for a review). According to the theory of event coding, action and perception codes share a common representational system (Herwig, 2015; Hommel, Müsseler, Aschersleben, \& Prinz, 2001). These authors assume bidirectional influences between perception and action planning or execution. In the visuomotor domain, O'Regan and Noë (2001) proposed that visual perception cannot be investigated outside sensorimotor contingencies between eye movements and changes in the retinal input. Interestingly, Burr, Ross, Binda, and Morrone (2011) have already investigated the issue of a close relationship between perception and ocular saccades (rapid shifts in gaze direction bringing the image of an object on the fovea). They briefly flashed a stimulus around the time of saccade and observed mislocalization of the stimulus in the direction of the saccadic target (Burr et al., 2011; see also Ross, Burr, \& Morrone, 1996). This phenomenon is called "saccadic compression" of visual space. Similar effects have been reported for time (Binda, Cicchini, Burr, \& Morrone, 2009) and number (Binda, Morrone, Ross, \& Burr, 2011). Crucially, these phenomena are only observed for a specific time window, around the onset ( \pm 100 milliseconds) of saccades, which supports the influence of eye movements on perceptual processing. Furthermore, similar time courses for these compression phenomena likely confirm the involvement of a common processing system for magnitudes, occurring in the intraparietal cortex, where transient shifts of receptive fields of neurons (i.e., remapping) are also observed during saccade planning (e.g., Wurtz, 2008). Such interweaved phenomena strongly suggest that the mere fact of generating a saccade influences perception. Spatiotemporal correlations do not, however, directly prove that action parameters (e.g., accelerating or slowing down, shortening or lengthening saccades) affect the perception of spatial, temporal, and numerical information. To demonstrate this, it is necessary to experimentally manipulate motor parameters and evaluate their effect on perception.

The double-step paradigm introduced by McLaughlin in 1967 is known to induce a rapid modification in saccadic amplitude, within 30-60 trials (e.g., Deubel, Wolf, \& Hauske, 1986). In this paradigm, the saccadic target jumps during eye movements so that the later land away from the new target position. Since visual acuity is drastically reduced during saccade execution (Bridgeman, Hendry, \& Stark, 1975), the target step is not perceived or hardly noticed most of the time. After several repetitions, the distance between the new target position and the saccade end point is progressively reduced by the oculomotor system so that the eyes land closer and closer to the new target position. This phenomenon, known as saccadic adaptation, manifests itself through longer or shorter saccadic amplitude depending on the direction (forward or backward) of repeated intrasaccadic target steps. Interestingly, modifications in object localization following such motor adaptation have been observed in several studies (e.g., Collins, Doré-Mazars, \& Lappe, 2007; Zimmermann \& Lappe, 2010; see Zimmermann \& Lappe, 2016, for a review). More precisely, when participants had to localize a visual object presented in the target vicinity prior to initiating an adapted saccade, objects were mislocalized in the direction of adaptation. Recently, Bosco, Lappe, and Fattori (2015) obtained intriguing results when increasing or decreasing the length of a visual target during saccades: this manipulation induced saccadic adaptation in the direction of object size modification-probably due to the shift of the stimulus 
gravity center. Bosco et al. (2015) found that perceived object size, estimated through grip aperture and verbal report, was affected in the same direction: Saccade amplitude and size estimation became smaller (or larger) when the postsaccadic object length was decreased (or increased). According to the authors, the changes in size perception would result from saccadic adaptation, the latter being due to the intrasaccadic modifications of object length. However, as noted by Valsecchi, Cassanello, Herwig, Rolfs, and Gegenfurtner (2020), it cannot be ruled out that the perceived size was only affected by the intrasaccadic change in object size. Indeed, Valsecchi and colleagues (2020; Valsecchi \& Gegenfurtner, 2016) found a recalibration of perceived size, but no modification of saccadic amplitudes, after systematically increasing or decreasing saccade target size during eye movements.

\section{The present study}

In order to specifically test whether motor changes influence perception, we measured the perceived size of a visual target before and after saccadic adaptation. Participants were asked to perform two embedded saccadic and perceptual tasks. Before the saccade, a test disk that varied in size was presented in the periphery. The disk was then replaced by a cross, which signaled to the participants that they had to make a saccade. After the eye movement, a fixed-size reference disk was foveally displayed, and participants had to judge whether it was larger (or not) than the test disk. Discrimination performance was assessed via psychometric functions obtained before and after backward saccadic adaptation from which we compared the points of subjective equality (PSEs), defined as the size of the presaccadic test disk that observer judged to be equal to the reference disk. If the magnitude of saccadic parameters is processed by a general system along the same continuous scale as other-perceptual-magnitudes, perceived object size should change in the same direction as saccadic adaptation. That is, perceived size would decrease. This should not be the case in a control group that did not experience saccadic adaptation (the target remained stationary during their saccades).

\section{Method}

\section{Participants}

Forty-two healthy participants, mostly students of Paris Descartes University, volunteered for the experiment (30 females, mean age $=24$ years). All had normal or corrected-tonormal vision, and none reported any mental or neurological disorder. They were naïve about the purpose of the study and signed an informed consent before the experiment. Twenty- eight participants were right-handed (according to the Edinburgh Handedness Inventory; Oldfield, 1971). Among the participants, 22 performed the saccadic adaptation experiment while the others were allocated to the control group. Procedures were in line with the declaration of Helsinki and were approved by the local ethics committee of Paris Descartes University ( ${ }^{\circ}$ CER-PD: 2018-62).

\section{Stimuli and material}

Participants were seated in a dimly lit room, $57 \mathrm{~cm}$ from a screen, with their head on a chin rest and head restraint. The stimuli, generated with the Psychophysics Toolbox for MATLAB (Brainard \& Vision, 1997; Pelli, 1997), were displayed on a 24.5-in. BENQ screen (ZOWIE XL-2540), with a refresh rate of $144 \mathrm{~Hz}$ and a spatial resolution of $1,920 \times 1,080$. They were displayed on a grey screen (luminance $17 \mathrm{~cd} / \mathrm{m}^{2}$ ). The reference (foveal) stimulus was a black disk with a diameter of $1.8^{\circ}$, whose contours were blurred by a cumulative Gaussian gradient covering $20 \%$ of the diameter (equally distributed on each side). We presented nine different test disks whose area was a ratio of the reference disk. There were two extreme $(0.4$ and 1.6) and seven intermediate ratios $(0.7,0.8,0.9,1,1.1,1.2,1.3)$. A black cross $\left(0.7^{\circ} \times 0.7^{\circ}\right.$, line width 2 pixels) was used as fixation point as well as saccadic target. Eye movements were recorded with an EyeLink ${ }^{\circledR}$ 1000 (SR Research, Ontario, Canada) sampling at $1000 \mathrm{~Hz}$ and with a spatial resolution of $0.05^{\circ}$.

\section{Procedure and design}

Participants in the adaptation group performed a first phase of preadaptation, a second phase of adaptation, and third phase of end adaptation (these conditions are further detailed below). One phase included 184 trials: each of the two extreme ratios was presented eight times, while the seven intermediate ratios were presented 24 times each. The experiment, which lasted about 90 minutes, began with 18 practice trials followed by 552 test trials. Each phase started with a calibration procedure during which subjects had to track a point successively presented at nine different locations around the screen. Fixation was checked automatically at the beginning of each trial: gaze had to be inside an invisible window of $1.5^{\circ} \times 1.5^{\circ}$ centered on the fixation cross for a trial to be started.

\section{Trial description}

The task design is illustrated in Fig. 1. Each trial of the first and third phases (preadaptation and end adaptation) began with a fixation cross displayed between $4^{\circ}$ and $7^{\circ}$ to the left of the center of the screen. After a random delay (500 to 700 $\mathrm{ms}$ ), the test disk appeared at $12^{\circ}$ to the right of the fixation cross on which participants were instructed to keep fixating 


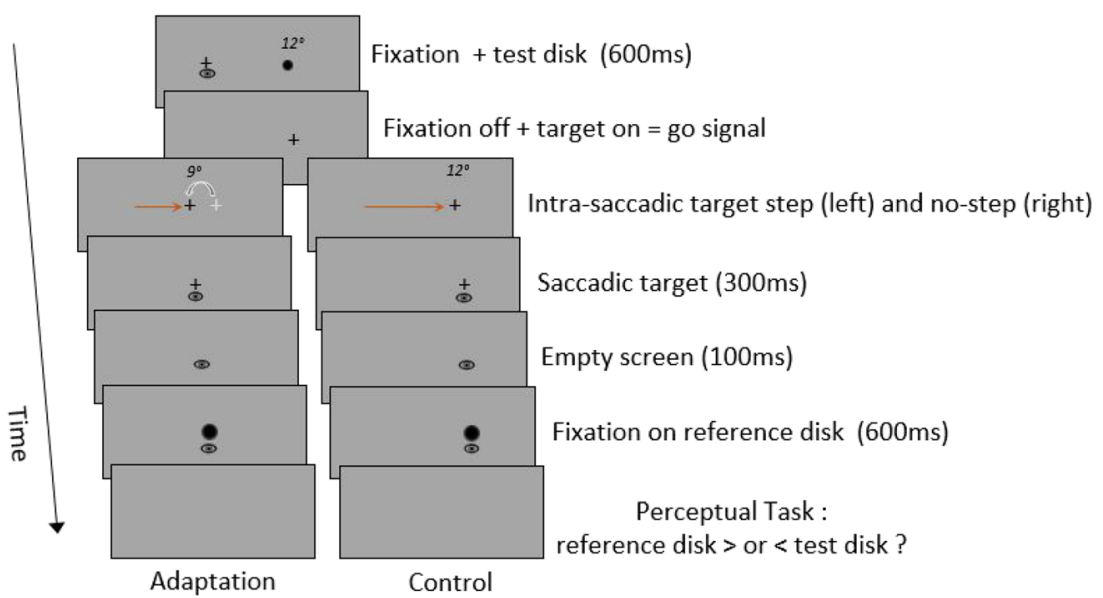

Fig. 1 Structure of a trial in Phase 3. A test disk (of varied size) was first presented at a $12^{\circ}$ eccentricity from a fixation cross. As soon as this disk was replaced by a cross and the fixation cross extinguished, participants made a saccade toward this cross, which was intrasaccadically shifted to $9^{\circ}$-in the backward adaptation group only (the presaccadic target position is materialized by the grey cross). Finally, participants compared the test and the reference disks and made their judgment with a manual response. Phase 1 was similar to Phase 3, except that no target shift occurred during saccades. Phase 2 was similar to Phase 3, without the perceptual task (see Fig. 1, Frame 1). After $600 \mathrm{~ms}$, the fixation cross disappeared, and the test disk was simultaneously replaced by a target cross (see Fig. 1, Frame 2). Subjects then had to execute their saccade toward this target only at the disappearance of the fixation cross. If saccade onset was detected before the extinction of the fixation cross or if saccade latency exceeded $800 \mathrm{~ms}$, the trial was aborted and presented again later on. After saccade detection, the target cross was displayed for $300 \mathrm{~ms}$ (see Fig. 1, Frame 4), followed by a $100-\mathrm{ms}$ empty screen (Fig. 1, Frame 5). Finally, the reference disk was displayed at the saccade target location for $600 \mathrm{~ms}$ (Fig. 1, Frame 6). After the extinction of this reference disk, participants could make their perceptual judgment by indicating whether it was larger (press on the up-arrow key) or smaller (press on the down-arrow key) than the presaccadic test disk (see Fig. 1, Frame 7). Five hundred milliseconds after the response, a new fixation cross signaled the beginning of the next trial. In the second phase (adaptation), the time course of the trials was the same, except that the reference disk was not displayed after the saccade, so that participants of both groups only performed the saccadic task.

\section{Backward adaptation group}

In the second (adaptation) and third (end adaptation) phases, the saccadic target, initially presented $12^{\circ}$ to the right of the fixation cross, was shifted toward the fixation cross as soon as a saccade was detected. In the adaptation phase, the target stepped by $1^{\circ}$ in the first 46 trials, $2^{\circ}$ in the next 46 trials, and $3^{\circ}$ in the 92 remaining trials. Throughout end-adaptation phase, the target always stepped by $3^{\circ}$, which means that at the end of the trials, the center of both the target cross and the reference disk was located $9^{\circ}$ to the right of the initial fixation.

\section{Control group}

The participants in the control group performed the same trials, with the same perceptual and saccadic tasks, except that the target was not shifted during saccades (in the endadaptation phase, the target cross and the reference disk were still located $12^{\circ}$ to the right of the initial fixation).

\section{Data analysis}

We recorded saccade latency, saccade amplitude, and the frequency of "smaller" responses for each ratio (i.e., the area of the test disk divided by the area of the reference disk). Latency was defined as the delay (in ms) between target appearance and saccade onset. Amplitude (in degrees of visual angle) corresponded to the distance covered by the eyes to reach the target. In off-line analyses, we used the EyeLink parser to identify saccade onset and offset (velocity threshold: $30 \%$, acceleration threshold: $8,000 \% \mathrm{~s}^{2}$ ). We plotted the proportion of trials per ratio in which the postsaccadic reference disk was judged smaller than the presaccadic test disk. Psychometric functions were parametrically fitted with a logistic function, using the fminsearch algorithm in MATLAB. Fit parameters (PSE; i.e., the size of the presaccadic test disk judged to be equal to the reference disk, and $95 \%$ confidence interval) were estimated with the maximum likelihood method. To estimate the discrimination sensitivity of each participant, we computed the just noticeable difference (JND) from the psychometric function. This parameter, related to the function steepness, defines the smallest difference perceived between two stimuli. The JND was calculated as follows: JND $=\left(x_{75}-x_{25}\right) / 2$, with $x_{.75}$ and $x_{.25}$ the ratios of the presaccadic test disk at which the probability to respond "reference disk smaller than the test 
disk" was $75 \%$ and $25 \%$, respectively. To assess the amount of saccadic adaptation, we computed a percentage change in saccade amplitude for each participant as follows: Percentage change in amplitude $=\left(\left(\right.\right.$ Amplitude $_{\text {first }}-$ Amplitude $\left._{\text {third }}\right) /$ Amplitude $\left._{\text {first }}\right) \times 100$. A similar formula was used to compute individual percentage changes in PSE: Percentage change in $\mathrm{PSE}=\left(\left(\mathrm{PSE}_{\text {first }}-\mathrm{PSE}_{\text {third }}\right) / \mathrm{PSE}_{\text {first }}\right) \times 100$.

Mixed analyses of variance (ANOVAs) were run on saccade latencies, amplitudes, JNDs, and PSEs, with group (backward adaptation vs. control) as a between-subjects factor, and phase (first vs. third) as a within-subjects factor.

Saccades landing more than $5^{\circ}$ away from the postsaccadic target were excluded from analyses (5\% and $3 \%$ of the data in the backward adaptation and control groups, respectively). After discarding saccades with latencies shorter than 80 milliseconds (9\% and $10 \%$ in the backward adaptation and control groups), we removed latency outliers (3\% of the data in each group) based on Tukey's rule (Carling, 2000). Overall, $17 \%$ and $16 \%$ of the data were discarded in the backward adaptation and control groups, respectively.

\section{Results}

\section{Saccade parameters and requirements}

First, we examined the characteristics (latency and amplitude) of the saccades recorded in the first and third phases. Mean saccade latencies (see Table 1) were not affected by group, $F(1,40)=1.63, p=.21$. The ANOVA revealed a significant main effect of phase, with latencies decreasing between the first and the third phase, which might reflect a training effect, $F(1,40)=50.2, p<.001$. The interaction was not significant, $F(1,40)=1.46, p=.23$.

For mean saccade amplitudes, the ANOVA revealed main effects of group, $F(1,40)=50.8, p<.001$, and phase, $F(1,40)$ $=331.7, p<.001$, as well as a significant interaction, $F(1,40)$ $=438, p<.001$, mainly due to the mean $2.20^{\circ}$ decrease in amplitude between the first and third phase in the backward adaptation group $(p<.001)$. There was no significant change

Table 1 Means \pm standard deviations of saccade amplitudes, latencies, JNDs, and PSEs, for both groups and phases

\begin{tabular}{lccccc}
\hline & \multicolumn{2}{c}{ Backward adaptation } & & \multicolumn{2}{c}{ Control: No adaptation } \\
\cline { 2 - 3 } \cline { 5 - 6 } \cline { 5 - 6 } & Phase 1 & Phase 3 & & Phase 1 & Phase 3 \\
\hline Latency (ms) & $235 \pm 97$ & $205 \pm 40$ & & $259 \pm 74$ & $218 \pm 44$ \\
Amplitude $\left({ }^{\circ}\right)$ & $11.85 \pm 0.5$ & $9.66 \pm 0.5$ & & $11.68 \pm 0.5$ & $11.89 \pm 0.5$ \\
JND & $0.11 \pm 0.05$ & $0.10 \pm 0.04$ & & $0.11 \pm 0.05$ & $0.10 \pm 0.04$ \\
PSE & $0.945 \pm 0.1$ & $0.981 \pm 0.1$ & & $0.988 \pm 0.1$ & $0.998 \pm 0.1$ \\
\hline
\end{tabular}

in amplitude in the control group $\left(\Delta_{\mathrm{Amp}}=0.21^{\circ}, p=.17\right)$, which was consistent with our expectations. Importantly for our purpose, no difference between groups in saccade amplitude was observed in the first phase $(p=.72)$. As expected, all participants in the backward adaptation group showed a consistent decrease in saccade amplitude. Their mean percentage change in saccade amplitude, which significantly differed from zero $(p<.001)$, was $18.5 \% \pm 3.33 \%$, with a minimum of $10.1 \%$ and a maximum of $23.8 \%$. Note that the target step from $12^{\circ}$ to $9^{\circ}$ represents a $25 \%$ shift from the initial target eccentricity. Therefore, backward adaptation was successfully induced in the experimental group.

Second, we verified that the perceived difficulty of the task was similar in both groups. Individual discrimination performance was defined by the JND (see Table 1). This measure, negatively correlated with individual sensitivity, provides an estimation of task difficulty for each subject. Mean JNDs were similar in the two groups: both had a mean JND of $0.11( \pm 0.05)$ in the first phase, and of $0.10( \pm 0.04)$ in the third phase. The main effect of phase was significant, $F(1,40)=6.25, p=.015$, indicating a very slight increase in discrimination performance. Neither the effect of group, $F(1,40)=0.22, p=.88$, nor the interaction between phase and group reached significance, $F(1$, 40) $=0.093, p=.76$.

Third, we ensured that the presaccadic test disk's size did not affect saccade amplitude. We performed an ANOVA on saccade amplitudes, with ratio $(0.4,0.7,0.8,0.9,1,1.1,1.2$, $1.3,1.6)$ as a within-subjects factor and group as a betweensubjects factor. This analysis was conducted on the first phase only, before any experimental manipulation had occurred. This ANOVA showed no effect of ratio on saccade amplitudes, $F(8,319)=0.43, p=.90$, nor interaction with the factor group, $F(8,319)=0.15, p=.997$.

\section{Possible influence of natural variations in saccade amplitude}

According to the ATOM theory, shorter saccades should be associated with a smaller perceived size (i.e., a larger PSE) and larger saccades with a larger perceived size (i.e., a smaller PSE). To ensure that natural variations in saccade amplitude did not affect size perception, we computed two PSEs, one for small saccades (<median amplitude) and one for large saccades (>median amplitude), in each participant. Table 2 shows the mean amplitudes and PSEs calculated for both amplitude sizes (small vs. large).

We conducted an ANOVA, with amplitude (small vs. large saccades) as a within-subjects factor and group (adaptation vs. control) as a between-subjects factor. Neither amplitude, $F(1$, $40)=0.081, p=.88$, nor group $F(1,40)=1.51, p=.32$, had a significant effect on PSEs. These data show that natural variations in saccade amplitude (independently of any 
Table 2 Means \pm standard deviations of saccade amplitudes and PSEs as a function of Amplitude (small vs. large saccades) for each group

\begin{tabular}{lrrrrr}
\hline & \multicolumn{2}{l}{ Backward adaptation } & & \multicolumn{2}{l}{ Control: No adaptation } \\
\cline { 2 - 3 } \cline { 5 - 6 } \cline { 5 - 6 } & $\begin{array}{l}\text { Small } \\
\text { saccades }\end{array}$ & $\begin{array}{l}\text { Large } \\
\text { saccades }\end{array}$ & & $\begin{array}{l}\text { Small } \\
\text { saccades }\end{array}$ & \multicolumn{1}{l}{$\begin{array}{l}\text { Large } \\
\text { saccades }\end{array}$} \\
\hline Amplitude $\left(^{\circ}\right)$ & $11.3 \pm 0.57$ & $12.5 \pm 0.51$ & $11.0 \pm 0.59$ & $12.3 \pm 0.41$ \\
PSE & $0.948 \pm 0.12$ & $0.943 \pm 0.11$ & $0.981 \pm 0.11$ & $0.991 \pm 0.12$ \\
\hline
\end{tabular}

experimental manipulation) are not sufficient to influence size perception.

\section{Effect of backward adaptation on size perception}

If saccadic adaptation (i.e., the reduction of saccade amplitude below the natural saccade hypometria) affects size perception (i.e., if a decrease in saccade amplitude induces a decrease in perceived size), an increase in PSE between the first and the third phase is then expected. Such an increase means that the presaccadic test disk has to be larger than the reference disk to be perceived as equal; in other words, the size of the test disk has been underestimated in comparison with the test disk in the preadaptation phase. The psychometric functions for one representative participant of the backward adaptation group are shown in Fig. 2 (left panel). A rightward shift of the psychometric function is visible in the third phase. This increase in PSE is reflected in a negative percentage change in PSE $(-7.22 \%$ for this subject; $-4.05 \% \pm 5.98 \%$ on average for the group). Furthermore, as showed in Fig. 3, 19 out of 22 participants displayed this expected pattern. The right panel of Fig. 2 shows the perceptual performance of one representative participant of the control group. No shift of the psychometric function was observed in the third phase (the corresponding percentage change was $1.92 \%$ ). The mean percentage change in the control group was $-1.22 \% \pm 6.84 \%$. In addition, the distribution of percentage change in PSE was symmetric around zero (see Fig. 3).

The ANOVA revealed a main effect of phase, $F(1,40)=$ $6.64, p=.014$ : Mean PSE increased between the first and the third phase, as shown in Table 1. There was no main effect of group, $F(1,40)=0.90, p=.35$, and the interaction between these two factors did not reach significance, $F(1,40)=2.30, p$ $=.14$. The lack of interaction is not critical because it does not actually take into account the difference between groups in baseline perception (i.e., PSE in first phase). It is then more relevant to assess the effect of phase in each group separately, by comparing the change in PSE to zero (i.e., no change in perception). The results actually showed a significant increase in PSE between phases in the adaptation group $\left(\Delta_{\mathrm{PSE}}=0.036\right)$, $t(19)=3.47, p=.002$. This increase is more than four times as large as the difference observed in the control group $\left(\Delta_{\mathrm{PSE}}=\right.$ $0.007), t(19)=0.64, p=.53$. This supports the idea that the perceptual change observed in the backward adaptation group was due to saccadic adaptation.

\section{Discussion}

The present experiment takes advantage of the saccadic system plasticity to test the impact of motor changes on objectsize perception. Following the saccadic adaptation induced by the double-step paradigm, participants showed the expected decrease in saccade amplitude. Saccadic adaptation was not observed among participants in the control group whose saccades toward a target remained stationary. Since latencies and JNDs (assessing subjective task difficulty) were similar in the

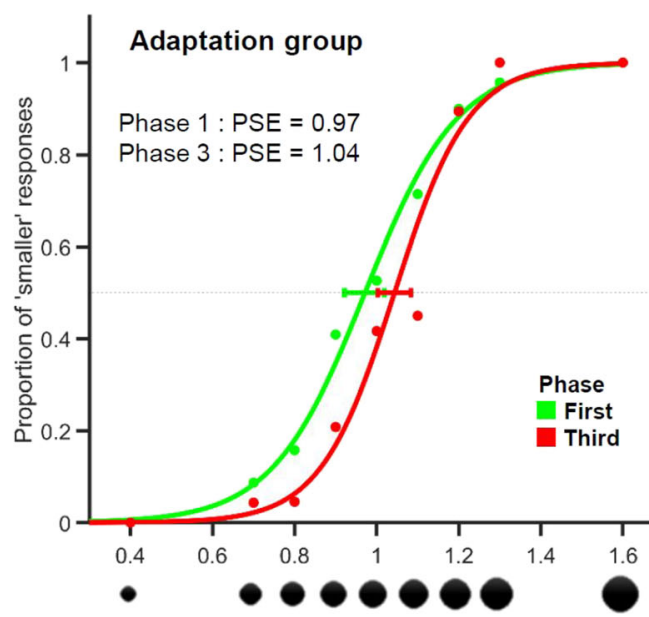

Fig. 2 Examples of individual psychometric functions in the first (green) and third (red) phases, obtained from the backward adaptation group (left panel) and control group (right panel). $Y$-axis: Proportion of "smaller" responses (to the question: "Is the second disk larger or smaller than the

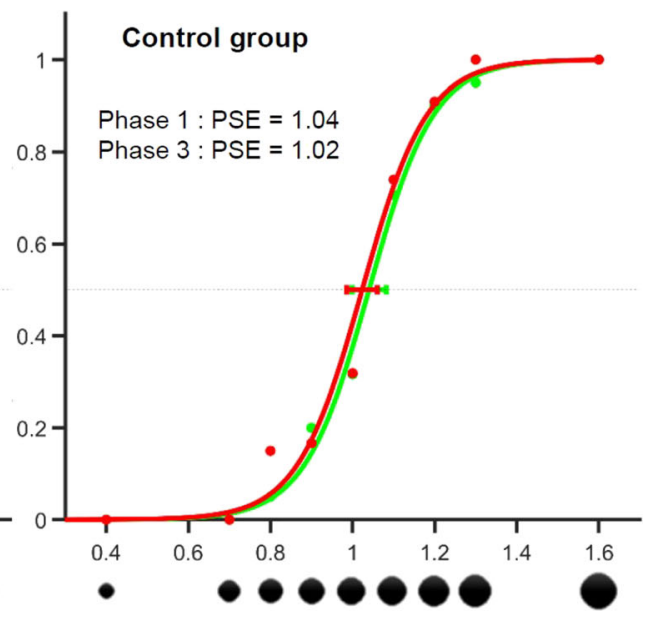

first one?") in the perceptual task. $X$-axis: Ratios of the test disk's size (variable) to the reference disk's size. Error bars indicate 95\% bootstrap confidence intervals 


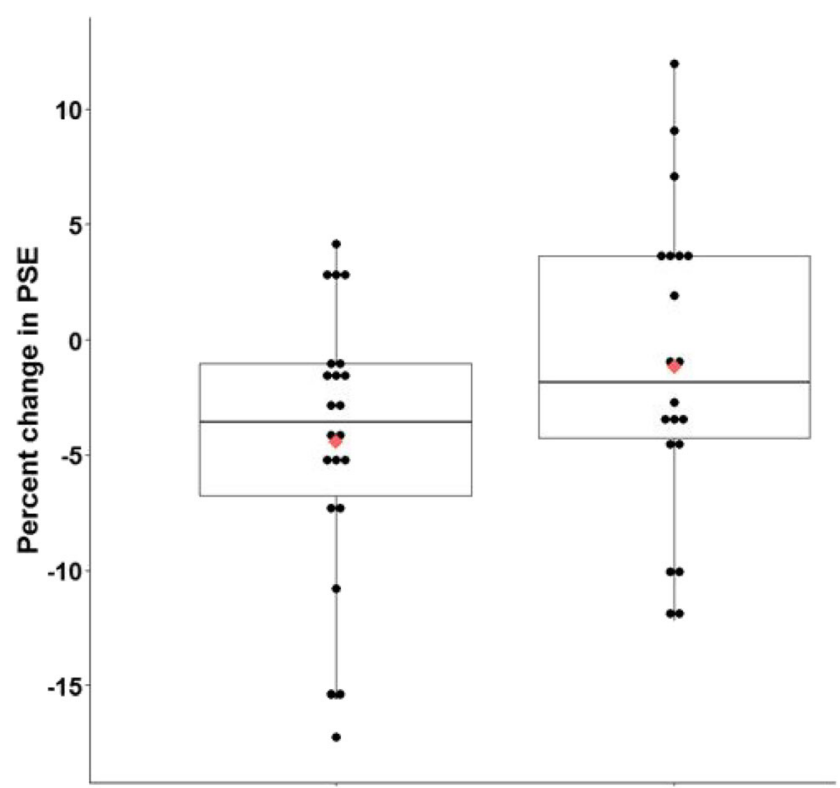

Fig. 3 Boxplots of percentage of changes in PSE in each group. Diamonds indicate the mean of the distributions, and each point represents one participant. A negative value corresponds to an increase in PSE from Phase 1 to Phase 3, meaning that in comparison with the baseline, the size of the presaccadic test disk was underestimated after backward saccadic adaptation

two groups, it can be concluded that the subsequent perceptual changes observed only in the backward adaptation group were due to the intrasaccadic target step.

In line with the ATOM theory, one would predict that perceived size would correlate with natural variations in saccade amplitudes. In the first phase, however, the PSEs did not differ for small and large saccade amplitudes for both groups (cf. Table 2). This shows that the range of natural variations in saccade amplitudes is too narrow to affect size perception. A significant decrease in perceived size was observed only after the double-step paradigm that reduced saccadic amplitudes below natural hypometria. It is, of course, still possible that size perception of peripheral objects depends on the amplitude of impending, regular (i.e., nonadapted) saccades. Our paradigm does not allow for a definitive answer to this question, which should be addressed in future experiments.

As predicted by our hypothesis, PSEs increased after saccadic adaptation: When compared with the baseline, the size of the presaccadic test disk presented in the saccadic adaptation field was underestimated. By contrast, PSEs did not change between the first and the third phases in the control group, indicating stable size perception throughout the experiment. This difference between groups was observed even though participants only made rightward saccades. Indeed, we could have obtained a larger effect size for saccades directed to the left as opposed to the right. This assumption is based on Irwin and Thomas's (2007) findings that showed that only saccades made to the left (vs. no saccade and saccade to the right) interfered with number comparison, a task that relies on the parietal cortex. The authors interpreted these results in terms of interference within the dorsal stream, which is involved in saccade generation and saccade execution.

Overall, our results corroborate the view that modifying an action parameter (decreasing saccade amplitude) affects the perception of a magnitude in the same direction (decreased perceived object size). Our conclusion complements the ATOM model (Bueti \& Walsh, 2009; Walsh, 2003), which predicts that modifying one perceptual dimension will affect the perception of another dimension in the same direction (i.e., more A-more B). What our study highlights is that action is not merely an output of the model, but rather one of the dimensions processed by the general system of magnitudes. Our findings extend studies showing that saccade generating affects numerical processing (Irwin \& Thomas, 2007) as well as the perception of stimuli presented around the time of saccades (Burr et al., 2011). They also complement studies focusing on the relation between grasping and perceptual magnitude processing (Badets \& Pesenti, 2010; Job, van Velzen, \& de Fockert, 2017; Seegelke \& Wühr, 2018).

Our protocol has the advantage of disambiguating the critical dimension (target location) that is needed to aim at the target from the object feature (its size) involved in the perceptual task. In the double-step paradigm, moving the target (i.e., changing its location during saccades) induces retinal errors (the retinal eccentricity of the target when the eye lands after saccades). The system progressively compensates for them by recalibrating its motor commands (Hopp \& Fuchs, 2004; Pélisson, Alahyane, Panouillères, \& Tilikete, 2010). These motor changes might be accompanied by changes in spatial maps, as indicated by the shift of perceived objects' location in the direction of saccadic adaptation (Collins et al., 2007; Garaas \& Pompluns, 2011; Zimmermann \& Lappe, 2010; see Zimmermann \& Lappe, 2016, for a recent review). Note that in these studies, the perceptual test measured spatial localization. In another recent study, Bosco et al. (2015) induced saccadic adaptation via a change in the size of the visual target during saccades. They observed a distortion in perceived size that they attributed to the motor change. In this size-based adaptation, object size was a critical parameter for participants to aim at the target, whereas such information is less relevant for classic double-step adaptation. A common feature of these studies is the finding of a change in the perception of the dimension relevant for saccade programming, whereas in our experiment, the visuomotor system only uses target localization to determine saccade amplitude and object size to perform the perceptual task. Indeed, we sought and managed to avoid potential effects related to the size of the test disk presented before saccades (as observed for hand movements, see Ren et al., 2011; Seegelke \& Wühr, 2018; Wühr \& Seegelke, 2018). To achieve this, we replaced the peripheral test disk with a different saccadic target (a cross) that was stepped during saccades. Moreover, we maintained the center of mass 
of both the presaccadic test disk and the target cross not only aligned, but also constant through trials (contrary to Bosco et al.'s, 2015, study, in which the center of mass was modified with the variations in object size). This was crucial because saccades are known to land on the center of mass of visual targets (He \& Kowler, 1991). Finally, anticipatory saccades whose latencies were shorter than 80 milliseconds were discarded from our analyses. Their planning is indeed the most likely to be influenced by the presentation of the test disk - provided that the disk size affects saccade programming. Most importantly, our statistical analyses confirmed that the size of the disk did not impact saccade amplitude (see the Requirements section). Therefore, our study is the first one to provide evidence that motor change itself (i.e., recalibrating saccade amplitude) leads to modification in size perception, even though this information is not critical for sensorimotor transformation.

In addition, our data can rule out the alternative hypothesis of distortions in the spatial map following saccadic adaptation. According to several studies, because saccadic adaptation modified object localization in the direction of adaptation (e.g., Collins et al., 2007; Zimmermann \& Lappe, 2010; see Zimmermann \& Lappe, 2016, for a recent review), it should distort the representation of visual space by changing retinal coordinates. If this was the case, then visual objects in the vicinity of the saccade target (such as the presaccadic test disks in our experiment) would have appeared closer to fixation following backward adaptation. Importantly, it is also known that there is a reduction in the apparent size of objects when they are seen in peripheral vision and that this reduction is correlated to the object eccentricity (Baldwin, Burleigh, Pepperell, \& Ruta, 2016; Bedell \& Johnson, 1984; Newsome, 1972). Therefore, following this rational, the test disks in our study would have appeared larger as a consequence of the reduced apparent eccentricity. Yet we observed a decrease in the perceived size, which is consistent with the ATOM theory.

A number of behavioral and neurological data have provided evidence for a common metric for magnitude processing and action control. For instance, Irwin and Thomas (2007), as previously mentioned, showed that saccades interfere with perceptual tasks involving the dorsal stream. In their protocol, participants had to perform either a parity or a number comparison task while making saccades at different eccentricities. They found that reaction times depended on eccentricity in the number comparison task. This task is known to rely on the dorsal stream and especially on the right parietal cortex (Dehaene, 1996; Fias, Lammertyn, Reynvoet, Dupont, \& Orban, 2003; Pinel, Dehaene, Riviere \& LeBihan, 2001), while the parity judgment task rather tends to rely on verbal processing in the ventral stream (Irwin \& Thomas, 2007). Besides, it has been shown that neural areas involved in the processing of quantities and in action control overlap in parietal brain regions (Bueti \& Walsh, 2009). Specifically, the intraparietal sulcus (IPS) and the prefrontal cortex of primates are involved in the processing of numerical, spatial, and temporal information (Duhamel, Colby, \& Goldberg, 1998) as well as in sensorimotor transformations (Gallivan \& Culham, 2015). The intraparietal region is also known for its role in both saccade programming and physical space processing (Knops, Thirion, Hubbard, Michel, \& Dehaene, 2009; Simon, Mangin, Cohen, Le Bihan, \& Dehaene, 2002). Interestingly, specific activation has been recently observed in the parietal cortex during saccadic adaptation (Gerardin, Miquée, Urquizar, \& Pélisson, 2012; Guillaume, Fuller, Srimal, \& Curtis, 2018; Panouillères, Habchi, et al., 2012; Panouillères, Neggers, et al., 2012; Pélisson, Habchi, Panouillères, Hernoux, \& Farnè, 2018). This overlap in the parietal lobe might explain the interference we observed between saccadic adaptation and size perception.

\section{Conclusion}

In summary, we showed that motor changes influence object size perception: Decreasing saccade amplitudes induces a decrease in the perceived size of a visual object presented at the saccade target location. The present study thus makes clear for the first time that action is an integral part of the magnitudes processed by the general system of magnitude proposed by Walsh (2003). It provides further evidence for two-way influences between spatial and motor maps and suggests the existence of common neural codes for action and perception.

Acknowledgements The authors warmly thank Anne Hillairet de Boisferon for helpful comments on an earlier version of the manuscript, Agnès Charvillat for her help with English, and Lara Kech for her contribution to data collection.

This research received funding from a scholarship from the Ministry of Research (A.P.) and a grant from the Agence Nationale pour la Recherche (ANR-18-CE28-0001) to C.P.

Open practices statement Raw data are available on Open Science Framework (https://osf.io/u2e3a/). The experiment was not preregistered.

\section{References}

Badets, A., \& Pesenti, M. (2010). Creating number semantics through finger movement perception. Cognition, 115(1), 46-53.

Baldwin, J., Burleigh, A., Pepperell, R., \& Ruta, N. (2016). The perceived size and shape of objects in peripheral vision. i-Perception, 7(4). https://doi.org/10.1177/2041669516661900

Bedell, H. E., \& Johnson, C. A. (1984). The perceived size of targets in the peripheral and central visual fields. Ophthalmic and Physiological Optics, 4(2), 123-131.

Binda, P., Cicchini, G. M., Burr, D. C., \& Morrone, M. C. (2009). Spatiotemporal distortions of visual perception at the time of saccades. Journal of Neuroscience, 29(42), 13147-13157. 
Binda, P., Morrone, M. C., Ross, J., \& Burr, D. C. (2011). Underestimation of perceived number at the time of saccades. Vision research, 51(1), 34-42.

Bosco, A., Lappe, M., \& Fattori, P. (2015). Adaptation of saccades and perceived size after trans-saccadic changes of object size. Journal of Neuroscience, 35(43), 14448-14456.

Brainard, D. H., \& Vision, S. (1997). The Psychophysics Toolbox. Spatial Vision, 10, 433-436.

Bridgeman, B., Hendry, D., \& Stark, L. (1975). Failure to detect displacement of the visual world during saccadic eye movements. Vision Research, 15(6), 719-722.

Bueti, D., \& Walsh, V. (2009). The parietal cortex and the representation of time, space, number and other magnitudes. Philosophical Transactions of the Royal Society B: Biological Sciences, 364(1525), 1831-1840.

Burr, D. C., Ross, J., Binda, P., \& Morrone, M. C. (2011). Saccades compress space, time, and number. In S. Dehaene \& E. Brannon (Eds.), Space, time and number in the brain (pp. 175-186). London, England: Academic Press.

Carling, K. (2000). Resistant outlier rules and the non-Gaussian case. Computational Statistics \& Data Analysis, 33(3), 249-258.

Collins, T., Doré-Mazars, K., \& Lappe, M. (2007). Motor space structures perceptual space: Evidence from human saccadic adaptation. Brain Research, 1172, 32-39.

Creem-Regehr, S. H., \& Kunz, B. R. (2010). Perception and action. Wiley Interdisciplinary Reviews: Cognitive Science, 1(6), 800-810.

Culham, J. C., \& Valyear, K. F. (2006). Human parietal cortex in action. Current Opinion in Neurobiology, 16(2), 205-212.

Dehaene, S. (1996). The organization of brain activations in number comparison: Event-related potentials and the additive-factors method. Journal of Cognitive Neuroscience, 8(1), 47-68.

Dehaene, S., Bossini, S., \& Giraux, P. (1993). The mental representation of parity and number magnitude. Journal of Experimental Psychology: General, 122(3), 371.

DeLong, A. J. (1981). Phenomenological space-time: Toward an experiential relativity. Science, 213(4508), 681-683.

Deubel, H., Wolf, W., \& Hauske, G. (1986). Adaptive gain control of saccadic eye movements. Human Neurobiology, 5(4), 245-253.

Dotan, D., \& Dehaene, S. (2013). How do we convert a number into a finger trajectory? Cognition, 129(3), 512-529.

Duhamel, J. R., Colby, C. L., \& Goldberg, M. E. (1998). Ventral intraparietal area of the macaque: Congruent visual and somatic response properties. Journal of Neurophysiology, 79(1), 126-136.

Fias, W., Lammertyn, J., Reynvoet, B., Dupont, P., \& Orban, G. A. (2003). Parietal representation of symbolic and nonsymbolic magnitude. Journal of Cognitive Neuroscience, 15(1), 47-56.

Fischer, M. (2003). Spatial representations in number processingEvidence from a pointing task. Visual Cognition, 10, 493-508.

Fischer, U., Fischer, M. H., Huber, S., Strauß, S., \& Moeller, K. (2018). The influence of number magnitude on continuous swiping movements. Journal of Numerical Cognition, 4(2), 297-316.

Fischer, M. H., Warlop, N., Hill, R. L., \& Fias, W. (2004). Oculomotor bias induced by number perception. Experimental Psychology, 51(2), 91-97.

Gallivan, J. P., \& Culham, J. C. (2015). Neural coding within human brain areas involved in actions. Current Opinion in Neurobiology, $33,141-149$.

Garaas, T. W., \& Pomplun, M. (2011). Distorted object perception following whole-field adaptation of saccadic eye movements. Journal of vision, 11(1), 2-2.

Gerardin, P., Miquée, A., Urquizar, C., \& Pélisson, D. (2012). Functional activation of the cerebral cortex related to sensorimotor adaptation of reactive and voluntary saccades. NeuroImage, 61(4), 1100-1112.

Guillaume, A., Fuller, J. R., Srimal, R., \& Curtis, C. E. (2018). Corticocerebellar network involved in saccade adaptation. Journal of Neurophysiology, 120(5), 2583-2594.
He, P., \& Kowler, E. (1991). Saccadic localization of eccentric forms. Journal of the Optical Society of America A, 8(2), 440-449.

Henik, A., \& Tzelgov, J. (1982). Is three greater than five: The relation between physical and semantic size in comparison tasks. Memory \& Cognition, 10(4), 389-395.

Hesse, P. N., Fiehler, K., \& Bremmer, F. (2016). SNARC effect in different effectors. Perception, 45(1/2), 180-195.

Herwig, A. (2015). Linking perception and action by structure or process? Toward an integrative perspective. Neuroscience \& Biobehavioral Reviews, 52, 105-116.

Hommel, B., Müsseler, J., Aschersleben, G., \& Prinz, W. (2001). The theory of event coding (TEC): A framework for perception and action planning. Behavioral and Brain Sciences, 24(5), 849-878.

Hopp, J. J., \& Fuchs, A. F. (2004). The characteristics and neuronal substrate of saccadic eye movement plasticity. Progress in Neurobiology, 72(1), 27-53.

Irwin, D. E., \& Thomas, L. E. (2007). The effect of saccades on number processing. Perception \& psychophysics, 69(3), 450-458.

Job, X. E., van Velzen, J., \& de Fockert, J. W. (2017). Grasp preparation modulates early visual processing of size and detection of local/ global stimulus features. Cortex, 96, 46-58.

Kadosh, R. C., Kadosh, K. C., Linden, D. E., Gevers, W., Berger, A., \& Henik, A. (2007). The brain locus of interaction between number and size: A combined functional magnetic resonance imaging and event-related potential study. Journal of Cognitive Neuroscience, 19(6), 957-970.

Knops, A., Thirion, B., Hubbard, E. M., Michel, V., \& Dehaene, S. (2009). Recruitment of an area involved in eye movements during mental arithmetic. Science, 324(5934), 1583-1585.

Markman, A. B., \& Brendl, C. M. (2005). Constraining theories of embodied cognition. Psychological Science, 16(1), 6-10.

McLaughlin, S. C. (1967). Parametric adjustment in saccadic eye movements. Perception \& Psychophysics, 2(8), 359-362.

Myachykov, A., Cangelosi, A., Ellis, R., \& Fischer, M. H. (2015). The oculomotor resonance effect in spatial-numerical mapping. Acta Psychologica, 161, 162-169.

Newsome, L. R. (1972). Visual angle and apparent size of objects in peripheral vision. Perception \& Psychophysics, 12, 300-304.

Oldfield, R. C. (1971). The assessment and analysis of handedness: The Edinburgh Inventory. Neuropsychologia, 9(1), 97-113.

O'Regan, J. K., \& Noë, A. (2001). A sensorimotor account of vision and visual consciousness. Behavioral and Brain Sciences, 24(5), 939 973.

Panouillères, M., Habchi, O., Gerardin, P., Salemme, R., Urquizar, C., Farne, A., \& Pélisson, D. (2012). A role for the parietal cortex in sensorimotor adaptation of saccades. Cerebral Cortex, 24(2), 304 314.

Panouillères, M., Neggers, S. F., Gutteling, T. P., Salemme, R., van der Stigchel, S., van der Geest, J. N., Frens, M.A. \& Pélisson, D. (2012). Transcranial magnetic stimulation and motor plasticity in human lateral cerebellum: Dual effect on saccadic adaptation. Human Brain Mapping, 33(7), 1512-1525.

Pélisson, D., Alahyane, N., Panouillères, M., \& Tilikete, C. (2010). Sensorimotor adaptation of saccadic eye movements. Neuroscience \& Biobehavioral Reviews, 34(8), 1103-1120.

Pélisson, D., Habchi, O., Panouillères, M. T., Hernoux, C., \& Farnè, A. (2018). A cortical substrate for the long-term memory of saccadic eye movements calibration. NeuroImage, 179, 348-356.

Pelli, D. G. (1997). The VideoToolbox software for visual psychophysics: Transforming numbers into movies, Spatial Vision, 10, 437442.

Piazza, M., \& Eger, E. (2016). Neural foundations and functional specificity of number representations. Neuropsychologia, 83, 257-273.

Pinel, P., Dehaene, S., Riviere, D., \& LeBihan, D. (2001). Modulation of parietal activation by semantic distance in a number comparison task. Neuroimage, 14(5), 1013-1026. 
Pressigout, A., \& Dore-Mazars, K. (2020). How does number magnitude influence temporal and spatial parameters of eye movements? Experimental Brain Research, 238(1), 101-109

Pressigout, A., Charvillat, A., Mersad, K., \& Doré-Mazars, K. (2018). Time dependency of the SNARC effect for different number formats: Evidence from saccadic responses. Psychological Research, 83, 1485-1495. https://doi.org/10.1007/s00426-018-1010-y

Prinz, W. (1990). A common coding approach to perception and action. In O. Neumann \& W. Prinz (Eds.), Relationships between perception and action (pp. 167-201). Berlin, Germany: Springer.

Ren, P., Nicholls, M. E., Ma, Y. Y., \& Chen, L. (2011). Size matters: Non-numerical magnitude affects the spatial coding of response. PLOS ONE, 6(8). https://doi.org/10.1371/journal.pone.0023553

Ross, J., Burr, D., \& Morrone, C. (1996). Suppression of the magnocellular pathway during saccades. Behavioural Brain Research, 80(1/2), 1-8.

Seegelke, C., \& Wühr, P. (2018). Compatibility between object size and response side in grasping: The left hand prefers smaller objects, the right hand prefers larger objects. PeerJ, 6, e6026.

Schwarz, W., \& Keus, I. M. (2004). Moving the eyes along the mental number line: Comparing SNARC effects with saccadic and manual responses. Perception \& Psychophysics, 66(4), 651-664.

Shin, Y. K., Proctor, R. W., \& Capaldi, E. J. (2010). A review of contemporary ideomotor theory. Psychological Bulletin, 136(6), 943.

Simon, O., Mangin, J. F., Cohen, L., Le Bihan, D., \& Dehaene, S. (2002). Topographical layout of hand, eye, calculation, and languagerelated areas in the human parietal lobe. Neuron, 33(3), 475-487.

Valsecchi, M., Cassanello, C., Herwig, A., Rolfs, M., \& Gegenfurtner, K.R. (2020). A comparison of the temporal and spatial properties of trans-saccadic perceptual recalibration and saccadic adaptation. Journal of Vision, 20(4), 2.

Valsecchi, M., \& Gegenfurtner, K. R. (2016). Dynamic re-calibration of perceived size in fovea and periphery through predictable size changes. Current Biology, 26(1), 59-63.

Walsh, V. (2003). A theory of magnitude: Common cortical metrics of time, space and quantity. Trends in Cognitive Sciences, 7(11), 483488.

Winter, B., Marghetis, T., \& Matlock, T. (2015). Of magnitudes and metaphors: Explaining cognitive interactions between space, time, and number. Cortex, 64, 209-224.

Wühr, P., \& Seegelke, C. (2018). Compatibility between physical stimulus size and left-right responses: Small is left and large is right. Journal of Cognition, 1(1), 17. https://doi.org/10.5334/joc.19

Wurtz, R. H. (2008). Neuronal mechanisms of visual stability. Vision Research, 48(20), 2070-2089.

Xuan, B., Zhang, D., He, S., \& Chen, X. (2007). Larger stimuli are judged to last longer. Journal of Vision, 7(10), 2-2.

Zimmermann, E., \& Lappe, M. (2010). Motor signals in visual localization. Journal of Vision, 10(6), 2-2.

Zimmermann, E., \& Lappe, M. (2016). Visual space constructed by saccade motor maps. Frontiers in Human Neuroscience, 10, 225. https://doi.org/10.3389/fnhum.2016.00225

Publisher's note Springer Nature remains neutral with regard to jurisdictional claims in published maps and institutional affiliations. 